\title{
Radionuclide imaging and therapy in a patient with coexistent diabetic foot syndrome and psoriatic arthritis
}

Marek M. Chojnowski', Beata Mrozikiewicz-Rakowska², Małgorzata Kobylecka' ${ }^{1}$, Leszek Czupryniak², Leszek Królicki ${ }^{1}$ Department of Nuclear Medicine, Medical University of Warsaw, Poland ${ }^{2}$ Department of Internal Medicine and Diabetology, Medical University of Warsaw, Poland

[Received 1 XII 2016; Accepted 29 III 2017]

\begin{abstract}
We report a case of 67-year-old man suffering from psoriatic arthritis, type 2 diabetes and diabetic foot syndrome. The patient presented symptoms of inflammation of the right ankle joint. Scintigraphic imaging with radiolabeled white blood cells was performed to differentiate whether the inflammation was related to psoriatic arthritis or diabetic foot syndrome. After revealing that, the inflammatory process was restricted only to the articular space of subtalar joint, the patient was diagnosed with exacerbation of psoriatic arthritis and qualified for radionuclide synovectomy. In patients with coexistent diabetic foot syndrome and inflammatory arthritis of the foot it is of vital importance to accurately differentiate these two conditions. We conclude that this can be potentially achieved with radiolabeled white blood cells scintigraphic imaging.
\end{abstract}

KEY words: diabetic foot syndrome, psoriatic arthritis, nuclear imaging, radionuclide synovectomy

Nucl Med Rev 2017; 20, 2: 101-102

\section{Background}

Diabetic foot syndrome (DFS) is a serious complication of diabetes mellitus (DM), affecting up to $10 \%$ of diabetic patients [1]. It could be potentially life-threatening and thus requires quick, accurate diagnosis and immediate treatment. This proves difficulty, since various forms of DFS (e.g. osteomyelitis or Charcot foot) have often the same initial clinical and radiological symptoms. The problem becomes even more challenging with the presence of a coexistent rheumatic disease affecting joints of the foot.

\section{Case report}

We describe a case of a 67-year-old patient suffering from psoriatic arthritis (PsA) for 22 years and type 2 DM complicated by Charcot neuroarthropathy for 15 years. The current treatment is oral methotrexate $25 \mathrm{mg}$ once a week and intensive insulinotherapy. The patient presented symptoms of inflammation of the right ankle joint, with swelling, tenderness and pain. In order to differentiate whether the clinical symptoms were the manifestation of acute Charcot

Correspondence to: Marek M. Chojnowski

Department of Nuclear Medicine

Medical University of Warsaw

Banacha 1a, 02-097 Warsaw, Poland neuroarthropathy or exacerbation of PSA, the patient was referred to the nuclear medicine department for scintigraphic evaluation.

The single photon emission computed tomography/ computed tomography (SPECT/CT) with technetium-99m labelled white blood

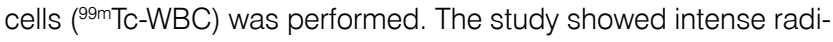
otracer uptake limited to the articular space of the right subtalar joint, with physiological ${ }^{99 \mathrm{~m} T c-W B C}$ distribution in the adjacent talus and calcaneus. In the CT scan, massive erosions and degeneration of subtalar joint articular surfaces were visible, with no signs of osteolysis or destruction of the cancellous bone (Figure 1A).

Based on these findings, inflammatory process involving bones was excluded. The patient was diagnosed with psoriatic arthritis of the right subtalar joint and subsequently qualified for radionuclide synovectomy. Under fluoroscopic guidance, the right subtalar joint was punctured from the lateral approach and $37 \mathrm{MBq}$ (mega Becquerels) of colloidal rhenium-186 sulphide were injected.

Within six months after radiosynovectomy, the clinical symptoms of joint inflammation subsided completely. The patient reported overall improvement and better life quality. Follow-up scintigraphic imaging with ${ }^{99 \mathrm{~m} T c-W B C}$ showed physiological distribution of the radiotracer, with no signs of active inflammatory process in the joints of the right foot (Figure 1B). Plain radiographs of the right foot showed marked improvement, with partial resolution of bone erosions. Twelve months after radiosynovectomy the patient remains symptom-free. 


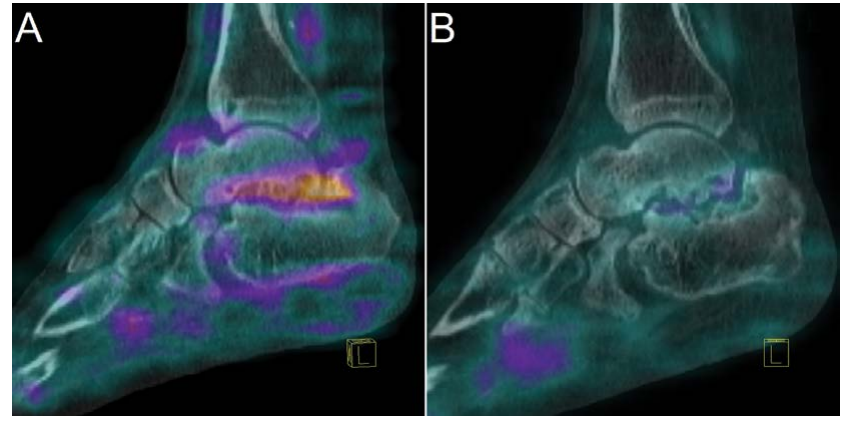

Figure 1. Initial SPECT/CT of the right foot shows intensive accumulation of radiolabeled white blood cells in the subtalar joint (A). Six months after radonuclide synovectomy there is only physiological distribution of radiolabeled white blood cells in the joints of right foot (B)

To the best of our knowledge this is the first report describing a patient with concomitant DFS and inflammatory arthritis of the foot successfully diagnosed and treated using radionuclide techniques.

\section{Discussion}

Patients with connective tissue diseases have a significantly increased risk of diabetes mellitus [2, 3]. Additionally, prolonged glucocorticoid therapy in these patients is an independent risk factor of developing steroid-induced diabetes. Populations of diabetic and rheumatic patients overlap and thus we may expect cases with concomitant DFS and arthritis of the foot. In such patients, initial clinical presentation of different foot conditions may be identical, and the results of both plain radiographs and MRI may be equivocal [4]. It is crucial to correctly identify the disease and start appropriate treatment, since misdiagnosis may have catastrophic consequences, e.g. intensifying immunosuppressive therapy in a patient with bacterial osteomyelitis. This is especially important in PsA where the involvement of the foot structures (enthesitis, tenosynovitis, arthritis) is common [5].

Diagnostic modalities of nuclear medicine, especially hybrid SPECT/CT imaging with ${ }^{99 m} \mathrm{Tc}-\mathrm{WBC}$ could be a valuable tool for diagnosing different inflammatory diseases of the foot. Although not specific enough to determine the exact cause of inflammation, they can precisely define the extent of the inflammatory process, indicating whether the soft tissues, bones or intra-articular space are affected. Moreover, SPECT/CT imaging can be used to qualify patients for minimally invasive intra-articular procedures like radionuclide synovectomy.

In conclusion, in patients with diabetic foot syndrome and coexisting inflammatory joint diseases, hybrid radionuclide imaging techniques might be considered the diagnostic method of choice. Further studies are required to determine their sensitivity and specificity in this particular clinical setting.

\section{Source of funding}

No funding was received for the presented work.

\section{Conflict of interest}

All the authors declare no conflicts of interest.

\section{Ethical standards}

All procedures were performed according to Helsinki Declaration.

\section{References}

1. Singh N, Armstrong DG, Lipsky BA. Preventing foot ulcers in patients with diabetes. JAMA. 2005; 293(2): 217-228, doi: 10.1001/jama.293.2.217, indexed in Pubmed: 15644549.

2. Solomon $\mathrm{DH}$, Love TJ, Canning $\mathrm{C}$, et al. Risk of diabetes among patients with rheumatoid arthritis, psoriatic arthritis and psoriasis. Ann Rheum Dis. 2010; 69(12): 2114-2117, doi: 10.1136/ard.2009.125476, indexed in Pubmed: 20584807

3. Mallbris L, Ritchlin CT, Ståhle M. Metabolic disorders in patients with psoriasis and psoriatic arthritis. Curr Rheumatol Rep. 2006; 8(5): 355-363, doi: 10.3899/jheum.120237, indexed in Pubmed: 16973109.

4. Graif M, Schweitzer ME, Deely D, et al. The septic versus nonseptic inflamed joint: MRI characteristics. Skeletal Radiol. 1999; 28(11): 616-620, indexed in Pubmed: 10591923.

5. Galluzzo E, Lischi DM, Taglione E, et al. Sonographic analysis of the ankle in patients with psoriatic arthritis. Scand J Rheumatol. 2000; 29(1): 52-55, doi: 10.1080/030097400750001806, indexed in Pubmed: 10722258. 